\title{
Sublattices of certain Coxeter lattices
}

\author{
par ANNE-MARIE BERGÉ et JACQUes MARTINET \\ À Georges Gras, pour ses soixante ans
}

RÉSUMÉ. Dans cet article, nous décrivons les sous-réseaux de certains réseaux de Coxeter, prolongeant les résultats de [Ber]. Notre description utilise des graphes.

ABStRaCt. In this paper, we describe the sublattices of some lattices, extending previous results of [Ber]. Our description makes intensive use of graphs.

\section{Introduction}

In his 1951 paper Extreme forms ([Cox]), the late Coxeter studied lattices $\Lambda$ which satisfy inclusions $\Lambda_{0} \subset \Lambda \subset \Lambda_{0}^{*}$ for some root lattice $\Lambda_{0}$. (As usual, $L^{*}$ stands for the dual of a lattice $L$, and a root lattice is an integral lattice generated by norm 2 vectors.) We shall more specially consider the root lattice $\Lambda_{0}=\mathbb{A}_{n}$, the section of $\mathbb{Z}^{n+1}$ endowed with its canonical basis $\mathcal{B}_{0}=\left(\varepsilon_{0}, \varepsilon_{1}, \ldots, \varepsilon_{n}\right)$ by the hyperplane $x_{0}+x_{1}+\cdots+x_{n}=0$. Then $\Lambda_{0}^{*} / \Lambda_{0}$ is cyclic of order $n+1$, so that there exists for every divisor $r$ of $n+1$ a well defined lattice $\mathbb{A}_{n}^{r}$ such that $\mathbb{A}_{n} \subset \mathbb{A}_{n}^{r} \subset \mathbb{A}_{n}^{*}$ and $\left[\mathbb{A}_{n}^{r}: \mathbb{A}_{n}\right]=r$. We denote by $\operatorname{Cox}_{n}\left(n\right.$ odd) the lattice $\mathbb{A}_{n}^{(n+1) / 2}$ scaled to the minimum which makes it integral and primitive. The lattices $\mathrm{Cox}_{n}$ are perfect lattices which have various curious properties. In particular, they are hollow in the sense of [Ber], and their minimum is odd whenever $n \equiv 3 \bmod 4$. This paper is a continuation of [Ber], which was devoted to the classification of crosssections of $\mathrm{Cox}_{n}$, but this time we consider sublattices of finite index (or more generally, of finite index in a cross-section). As [Ber], our paper makes intensive use of graphs to describe the possible sublattices of $\operatorname{Cox}_{n}$.

As in [Ber], Euclidean properties play only a modest rôle: the minimal vectors of $\mathbb{A}_{n}^{*}$ are vectors $\pm v_{0}, \pm v_{1}, \ldots, \pm v_{n}$ where the $v_{i}$ satisfy the unique (up to proportionality) dependence relation $v_{0}+v_{1}+\cdots+v_{n}=0$. We set

$$
S=\left\{v_{i}+v_{j}, 0 \leq i<j \leq n\right\} .
$$

Manuscrit reçu le 15 juin 2004.

Mots clefs. Eutactic lattices, spherical designs, kissing number, bipartite graphs. 
The lattice $\operatorname{Cox}_{n}$ is generated by $S$, which is actually the set of its minimal vectors (up to sign). We then essentially deal with submodules of the $\mathbb{Z}$-module generated by the $v_{i}$ : the Euclidean structure is only used in the last section where we consider some spherical 2-designs related to $\operatorname{Cox}_{n}$ (this is the property called strong eutaxy in [Ven]).

Notation. We denote by $E$ the span of the $v_{i}$, assuming that $n=\operatorname{dim} E \geq 5$, and of course that $n$ is odd (for the notation $\operatorname{Cox}_{n}$ to make sense). We denote by $L$ (resp. $M$ ) the $\mathbb{Z}$-module generated in $E$ by $v_{0}, \ldots, v_{n}$ (resp. by $\left.S\right)$. Note that $[L: M]=2$.

In Section 1, we characterize in terms of graphs the submodules $N$ of $L$ generated by subsets of $S$. We then determine in Section 2 the structure of the quotient modules $L / N$ and $M / N$. Finally, Section 3 is "the Euclidean section". We construct here a new infinite sequence of strongly eutactic sublattices of $\operatorname{Cox}_{n}$ having the same minimum.

\section{Graph of subsets of $S$}

Definition 2.1. With a subset $\mathcal{B}$ of $S$ we associate the following graph $\Gamma_{\mathcal{B}}$. Its vertex set is $V=\{0,1, \cdots, n\}$, and $i j$ is an edge of $\Gamma_{\mathcal{B}}$ if and only if $v_{i}+v_{j}$ lies in $\mathcal{B}$.

In order to study such a graph, some more notation is needed.

Notation. For every graph $G$ with vertex set $V_{G} \subset V$, put

$$
\begin{gathered}
e_{G}=\sum_{i \in V_{G}} v_{i}, \\
\mathcal{B}_{G}=\left\{e_{i j}=v_{i}+v_{j}, i j \text { edge of } G\right\},
\end{gathered}
$$

and

$$
L_{G}=\left\langle v_{i}, i \in V_{G}\right\rangle \quad \text { and } \quad M_{G}=\left\langle\mathcal{B}_{G}\right\rangle \subset L_{G},
$$

where for a subset $X$ of $E$, the notation $\langle X\rangle$ stands for the $\mathbb{Z}$-module generated by $X$.

If $G$ is a tree with bipartition $\left(V_{0}, V_{1}\right)$, we define the excess of $G$ to be

$$
d_{G}=|| V_{1}|-| V_{0}|| \text {. }
$$

In the case of an isolated vertex $G=\{i\}, d_{G}=1$.

[Recall that a tree is a connected graph without cycle; two vertices $i$ and $j$ of the tree are in the same vertex set $V_{k},(k=0,1)$, if the length of the path from $i$ to $j$ is even; see [Bo].]

Eventually, we say that two graphs $G$ and $G^{\prime}$ with the same vertex set are equivalent if the corresponding modules $M_{G}$ and $M_{G^{\prime}}$ coincide. 
Theorem 2.2. Let $\Gamma$ be a graph with vertex set $V=\{0,1, \ldots, n\}$ and $p \leq n$ edges, and let

$$
\Gamma=\bigcup_{C \in \mathcal{C}} C
$$

be its partition into connected components. Then the $p$ vectors $e_{i j}=v_{i}+v_{j}$, ij edge of $\Gamma$, are linearly independent if and only if the following conditions hold:

(1) every $C \in \mathcal{C}$ contains at most one odd cycle, and no even cycle;

(2) there exists exactly $n+1-p$ trees in $\mathcal{C}$;

(3) at least one of the trees has a strictly positive excess.

Before proving the theorem, let us discuss the modules associated with the type of subgraphs occurring in its statement. It may be convenient to use some canonical graphs. The shapes we have in mind (see Figure 1) are those of a kite (triangle linked to a path), of a double star (two stars with adjacent centres) or of a shooting star (a star linked to an even path). (A star may reduce to a single vertex.)
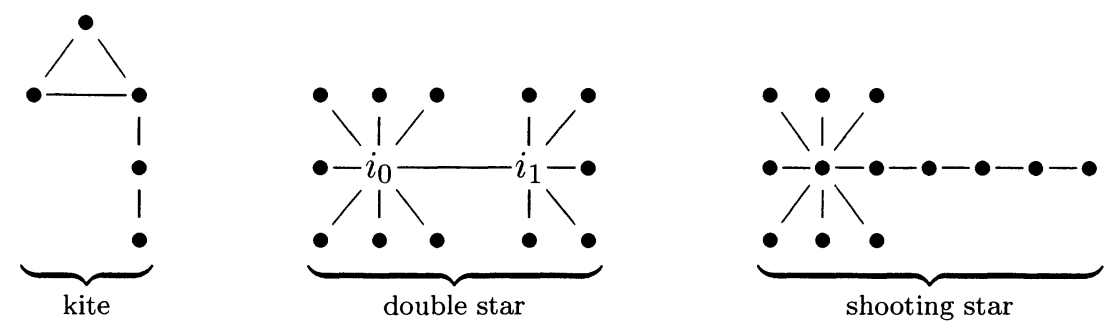

Figure 1

Our basic tool is the following, where we keep the notation $e_{i j}=v_{i}+v_{j}$.

Lemma 2.3. Let $i_{0} i_{1} i_{2} \ldots i_{m-1} i_{m}$ be a walk of length $m$ in $\Gamma$ (a cycle if $i_{m}=i_{0}$, a path otherwise). Then

$$
v_{i_{0}}+(-1)^{m-1} v_{i_{m}}=e_{i_{0} i_{1}}-e_{i_{1} i_{2}}+\cdots+(-1)^{m-1} e_{i_{m-1} i_{m}} .
$$

Proof. Clear.

Proposition 2.4. Let $C$ be a connected graph with vertex set $V_{C}$ strictly included in $V$, containing one odd cycle and no even cycle. Then the following holds for the set $\mathcal{B}_{C}=\left\{e_{i j}\right.$, ij edge of $\left.C\right\}$, its $\mathbb{Z}$-span $M_{C}$, and the vector $e_{C}=\sum_{i \in V_{C}} v_{i}$.

(1) For all vertices $i, j$ of $C$, the vectors $v_{i} \pm v_{j}$ belong to $M_{C}$.

(2) $C$ is equivalent to any kite with vertex set $V_{C}$.

(3) $M_{C}$ admits $\mathcal{B}_{C}$ as a $\mathbb{Z}$-basis, and $\operatorname{rank}\left(M_{C}\right)=|C|$.

(4) For all $i \in V_{C}$, the vector $2 v_{i}$ is an indivisible element of $M_{C}$.

(5) The vector $e_{C}$ belongs to $M_{C}$ if and only if $|C|$ is even. 
Proof. Since $C$ contains an odd cycle, two vertices $i$ and $j$ are joined by a path of odd length and by a path of even length (one of them including the cycle). Assertion 1 follows by applying Lemma 2.3 to these paths. This implies that $M_{C}=\left\langle e_{i j},(i, j) \in V_{C} \times V_{C}\right\rangle$ only depends on its vertex set; assertion 2 follows immediately. In particular, $M_{C}$ contains the vectors $2 v_{i}, i \in V_{C}$, and from $2 L_{C} \subset M_{C} \subset L_{C}$ it follows that $\operatorname{rank}\left(M_{C}\right)=$ $\operatorname{rank}\left(L_{C}\right)=|C|$ (because $|C|<n+1$ ). Since $C$ contains a unique cycle, $\mathcal{B}_{C}$ has $|C|$ elements, and therefore is a $\mathbb{Z}$-basis for its span. Let $i_{C}$ be a vertex of the cycle $\gamma$ contained in $C$. By Lemma 2.3, we obtain $2 v_{i_{C}}=$ $\sum_{j k \text { edges of } \gamma} \pm e_{j k}$, where the $e_{j k}$ belong to the basis $\mathcal{B}_{C}$ for $M_{C}$; thus $2 v_{i_{C}}$ is a primitive element of $M_{C}$, and by assertion 1 , this extends to all vertices of $C$. Eventually, from assertion 1 again it follows that the vector $e_{C}$ is congruent to $|C| v_{i_{C}}$ modulo $M_{C}$, and the fifth assertion results from the fourth one.

Proposition 2.5. Let $C$ be a non-trivial tree with vertex set $V_{C} \subset V$, bipartition $\left(V_{0}, V_{1}\right)\left(\left|V_{1}\right| \geq\left|V_{0}\right|\right)$, and excess $d_{C}=\left|V_{1}\right|-\left|V_{0}\right|$. For $i \in V_{C}$, define $\sigma(i) \in\{0,1\}$ by $i \in V_{\sigma(i)}$.

(1) For all $i, j$ in $V_{C}$ we have $(-1)^{\sigma(j)} v_{j} \equiv(-1)^{\sigma(i)} v_{i} \bmod M_{C}$.

(2) $C$ is equivalent to a double star with the same bipartition.

(3) $\mathcal{B}_{C}$ is a basis for $M_{C}$ if and only if $\left(|C|, d_{C}\right) \neq(n+1,0)$.

(4) If $\left(|C|, d_{C}\right) \neq(n+1,0)$, for all $i \in V_{C}$ there exists an indivisible element $w_{i} \in M_{C}$ such that $d_{C} v_{i}=w_{i}-(-1)^{\sigma(i)} e_{C}$.

[In the case of an isolated vertex $C=\{i\}$, the relation of assertion 4 is still valid with $w_{i}=0\left(\right.$ and $\left.d_{C}=1\right)$.]

Proof. Let $i, j \in V_{C}$. Lemma 2.3 applied to the path $i \leftrightarrow j$, whose length is congruent to $\sigma(i)-\sigma(j)$ modulo 2, proves assertion 1. Choose a pair $\left(i_{0}, i_{1}\right) \in V_{0} \times V_{1}$ of neighbours in $C$, and let $\mu_{1}$ (resp. $\left.\mu_{0}\right)$ be the maximal length of the paths $i_{0} \leftrightarrow j, j \in V_{1}$ (resp. $i_{1} \leftrightarrow i, i \in V_{0}$ ). We reduce first $\mu_{1}$, and then $\mu_{0}$, to be equal to 1 , by successive applications of the following lemma:

Lemma 2.6. Let $i j k \ell \subset C$ be a path of length 3 . Then replacing the edge $k \ell$ by the edge $i \ell$ we obtain a tree equivalent to $C$.

Proof of Lemma 2.6. By substituting $v_{i}+v_{\ell}=e_{i j}-e_{j k}+e_{k \ell}$ to $e_{k \ell}$ in $\mathcal{B}_{C}$ we obtain a new generator system for $M_{C}=\left\langle\mathcal{B}_{C}\right\rangle$.

For the remaining assertions, only depending on the bipartition and on the module $M_{C}$, we may suppose that $C$ is a double star with edges $i_{0} j$ and $i_{1} i,(i, j) \in V_{0} \times V_{1}$. The discussion of the linear independence of the $|C|-1$ vectors of $\mathcal{B}_{C}$ is then straightforward. For assertion 4 we may restrict ourselves to the case $i=i_{0}$ (see assertion 1$)$. We then have $e_{C}+d_{C} v_{i_{0}}=w_{i_{0}}$ 
with

$$
w_{i_{0}}=\sum_{x \in \mathcal{B}_{C}} x+\left(1-\left|V_{0}\right|\right) e_{i_{0} i_{1}} \in M_{C} .
$$

Note that at least one coefficient of $w_{i_{0}}$ on $\mathcal{B}_{C}$ is equal to 1 . Thus, if $\mathcal{B}_{C}$ is a basis for $M_{C}, w_{i_{0}}$ is primitive as required. This completes the proof of Proposition 2.5.

Proof of Theorem 2.2. In the case when $\Gamma$ is a tree, this results from Proposition 2.5. From now on we discard this case. The partition of $\Gamma$ gives rise to a partition $\mathcal{B}_{\Gamma}=\cup \mathcal{B}_{C}$ and to a sum $M_{\Gamma}=\sum M_{C}$ (for every $C \in \mathcal{C}, \mathcal{B}_{C}$ is the set of vectors $v_{i}+v_{j}$, ij edge of $C$, and $M_{C}$ is its span).

First note that if the vectors of $\mathcal{B}_{\Gamma}$ are linearly independent, $\Gamma$ contains no even cycle (otherwise, Lemma 2.3 applied to this cycle would provide a dependence relation between them). In the following we suppose that this condition holds. We now discuss the number $k_{C}$ of cycles contained in a given component $C \in \mathcal{C}$. Such a component has $\left|\mathcal{B}_{C}\right|=|C|+k_{C}-1$ edges; on the other hand, the inclusion $M_{C} \subset L_{C}$ implies $\mathrm{rk} M_{C} \leq|C|$. We then have the equivalences

$$
\mathcal{B}_{C} \text { is a basis for } M_{C} \Longleftrightarrow \operatorname{rk} M_{C}=\left|\mathcal{B}_{C}\right| \Longleftrightarrow k_{C}=0 \text { or } 1
$$

(the last equivalence makes use of Propositions 2.4 and 2.5). From now on, we assume $k_{C}=0$ or 1 for each $C \in \mathcal{C}$, i.e. that condition (1) of Theorem 2.2 holds. Then $\sum_{C}\left(1-k_{C}\right)=-\left|\mathcal{B}_{\Gamma}\right|+|V|$ is the number of trees in $\mathcal{C}$, as asserted in (2).

Now, $\mathcal{B}_{\Gamma}$ will be a basis for its span $M_{\Gamma}=\sum M_{C}$ if and only if this sum is direct. Since any dependence relation between the $v_{i}$ is proportional to $v_{0}+v_{1}+\cdots+v_{n}=0$, we have, for $\left(x_{C}\right) \in \prod_{C} M_{C}$, the equivalence

$$
\sum_{C} x_{C}=0 \Longleftrightarrow \exists a \in \mathbb{R} \text { such that } \forall C \in \mathcal{C}, x_{C}=a e_{C} .
$$

So, the sum $M_{\Gamma}=\sum M_{C}$ is direct if and only if there exists $C \in \mathcal{C}$ such that $e_{C}$ does not belong to the space $\mathbb{R} M_{C}$. Using the propositions above, one sees that this condition is equivalent to condition (3) of Theorem 2.2. This completes the proof of Theorem 2.2.

Remark 2.7. It can be convenient to use, as a reference tree, a shooting star instead of a double star. The degree of the centre of the star is then equal to $d+1$, where $d$ is the excess of the tree. In particular, the shooting star is a path if and only if $d=0$ or 1 .

\section{Quotient structures.}

In this section we assume that $\mathcal{B} \subset S$ is a basis for $E$ and we will describe the additive structures of the quotient groups $L / M_{\mathcal{B}}$ and $M / M_{\mathcal{B}}$, using the partition $\Gamma_{\mathcal{B}}=\bigcup_{C \in \mathcal{C}} C$ into components. By Theorem 2.2, there is a unique tree $T \in \mathcal{C}$, whose excess $d \geq 1$ is called excess of $\mathcal{B}$. The indices $\left[L: M_{\mathcal{B}}\right]$ 
and $\left[M: M_{\mathcal{B}}\right]$ only depend on the number of the components and on the excess of $\mathcal{B}$, but the actual structures also depends on the type of the graph:

Definition 3.1. The basis $\mathcal{B}$ is of odd type if its graph $\Gamma_{\mathcal{B}}$ contains a component $C$ with $|C|$ odd, and of even type if there is no such component.

Remark 3.2. Note that the type, as well as the number of components and the excess of the basis, may depend of the choice of the basis $\mathcal{B}$ for a given submodule $N$ of $M$. In particular, certain odd types can be reduced to even types: a graph $\Gamma_{\mathcal{B}}=T \cup C_{0} \cup G$ where $G$ is a union of kites of even order and $T$ a tree of excess 1 is equivalent to the graph obtained by replacing $T \cup C_{0}$ by a tree of excess 2 (see Figure 2).
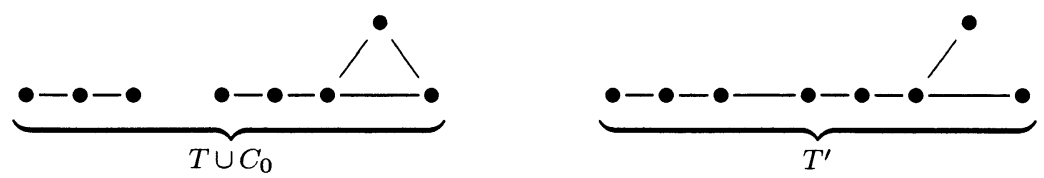

Figure 2

Theorem 3.3. Let $\mathcal{B}$ be a basis for $E$ contained in $S$, let $d \geq 1$ be its excess and let $c=|\mathcal{C}|$ be the number of components of its graph. Put $A=\mathbb{Z} / 2 d \mathbb{Z}$ (resp. $\mathbb{Z} / d \mathbb{Z} \times \mathbb{Z} / 2 \mathbb{Z}$ ) if $\mathcal{B}$ is of odd (resp. even) type. Then

(1) the group $L / M_{\mathcal{B}}$ is isomorphic to $A \times(\mathbb{Z} / 2 \mathbb{Z})^{c-2}$;

(2) the group $M / M_{\mathcal{B}}$ has order $d 2^{c-2}$ and is isomorphic to $\mathbb{Z} /(d / 2) \mathbb{Z}$ if $c=1$, and to $A \times(\mathbb{Z} / 2 \mathbb{Z})^{c-3}$ if $c \geq 2$.

Proof. To lighten the notation, we assume $\mathcal{B}$ of odd type, leaving to the reader the few changes needed for the even case. Let $\mathcal{C}=\{T\} \cup \mathcal{O} \cup \mathcal{E}$ be the set of components of the graph of $\mathcal{B}$, where $T$ denotes the tree, and $\mathcal{E}$ (resp. $\mathcal{O}$ ) denotes the set of components $C \neq T$ of even (resp. odd) order $|C|$. Let us fix a component $C_{0} \in \mathcal{O}$, and vertices $i_{T} \in V_{1}$ (the biggest vertex class of the tree) and $i_{C} \in V_{C}$ for each $C \neq T$.

Let $x=\sum_{i=0}^{i=n} a_{i} v_{i}$ be a vector of $L$, with $\left(a_{i}\right) \in \mathbb{Z}^{n+1}$ well defined up to translation. Put $A_{T}=-\sum_{i \in V_{T}}(-1)^{\sigma(i)} a_{i}$ (with $\sigma$ as in Proposition 2.5), and $A_{C}=\sum_{i \in V_{C}} a_{i}$ for $C \neq T$. We consider the following integers $\left(b_{C}\right)_{C \neq C_{0}}$ :

$$
b_{T}=A_{T}+d A_{C_{0}}, \quad b_{C}=A_{C} \text { for } C \in \mathcal{E}, \quad b_{C}=A_{C}-A_{C_{0}} \text { for } C \in \mathcal{O} .
$$

[Note that, under the translation $a_{i} \mapsto a_{i}+1, b_{C}$ is invariant modulo $2 d$ (resp. 2) for $C=T$ (resp. $C \neq T$ ).] By Propositions 2.4 and 2.5, and using in particular the relation $d v_{i T}=w_{T}-\sum_{C \neq T} e_{C}$ for some $w_{T} \in M_{T}$, we obtain:

$$
x \equiv A_{T} v_{i_{T}}+\sum_{C \neq T} A_{C} v_{i_{C}} \equiv y \quad \bmod M_{\mathcal{B}}
$$


with $y=\frac{b_{T}}{2 d}\left(2 w_{T}-2 \sum_{C \neq T} e_{C}\right)+\sum_{C \neq T, C_{0}} \frac{b_{C}}{2} 2 v_{i_{C}}$. Since the vectors $2 w_{T}-2 e_{C_{0}} \in M_{T} \oplus M_{C_{0}}$ and $2 v_{i_{C}} \in M_{C}\left(C \neq T, C_{0}\right)$ are indivisible, $y$ belongs to the module $M_{\mathcal{B}}=\left(M_{T} \oplus M_{C_{0}}\right) \oplus_{C \neq T, C_{0}} M_{C}$ if and only if $\frac{b_{T}}{2 d}$ and, for $C \neq T, C_{0}, \frac{b_{C}}{2}$ are integers. Let us denote by $\widetilde{a}$ (resp. $\bar{a}$ ) the residue class modulo $2 d$ (resp. 2) of an integer $a$. Then, the map

$$
\phi: x \mapsto\left(\widetilde{b_{T}},\left(\overline{b_{C}}\right)_{C \neq T, C_{0}}\right)
$$

from $L$ to $\mathbb{Z} / 2 d \mathbb{Z} \times(\mathbb{Z} / 2 \mathbb{Z})^{c-2}$ induces an additive isomorphism $L / M_{\mathcal{B}} \simeq \mathbb{Z} / 2 d \mathbb{Z} \times(\mathbb{Z} / 2 \mathbb{Z})^{c-2}$ as required for assertion 1 .

To prove assertion 2 we only need to describe the image $\phi(M)$ in $\mathbb{Z} / 2 d \mathbb{Z} \times(\mathbb{Z} / 2 \mathbb{Z})^{c-2}$ of the submodule

$$
M=\left\{\sum_{i} a_{i} v_{i} \in L \text { such that } \sum_{i} \overline{a_{i}}=\overline{0}\right\} .
$$

With the notation of the beginning of this proof, we have

$$
\begin{aligned}
\sum_{i} \overline{a_{i}} & =\sum_{C \in \mathcal{C}} \overline{A_{C}}=\left(\overline{b_{T}}-d \overline{A_{C_{0}}}\right)+\left(\sum_{C \in \mathcal{E}} \overline{b_{C}}\right)+\overline{A_{C_{0}}}+\sum_{C \in \mathcal{O} \backslash C_{0}}\left(\overline{b_{C}}+\overline{A_{C_{0}}}\right) \\
& =\sum_{C \neq C_{0}} \overline{b_{C}},
\end{aligned}
$$

because, since $\sum_{C}|C|=\left(2\left|V_{1}\right|-d\right)+\sum_{C \neq T}|C|=n+1$ is even, so is $|\mathcal{O}|+d$. The canonical homomorphism $f: \mathbb{Z} / 2 d \mathbb{Z} \rightarrow \mathbb{Z} / 2 \mathbb{Z}$ gives rise to a homomorphism $\tau:\left(u_{C}\right)_{C \neq C_{0}} \mapsto f\left(u_{T}\right)+\sum_{C \neq C_{0}, T} u_{C}$ of $\mathbb{Z} / 2 d \mathbb{Z} \times(\mathbb{Z} / 2 \mathbb{Z})^{c-2}$ onto $\mathbb{Z} / 2 \mathbb{Z}$. We can now state that the isomorphism $L / M_{\mathcal{B}} \simeq \mathbb{Z} / 2 d \mathbb{Z} \times$ $(\mathbb{Z} / 2 \mathbb{Z})^{c-2}$ gives rise to an exact sequence

$$
0 \longrightarrow M / M_{\mathcal{B}} \longrightarrow \mathbb{Z} / 2 d \mathbb{Z} \times(\mathbb{Z} / 2 \mathbb{Z})^{c-2} \stackrel{\tau}{\longrightarrow} \mathbb{Z} / 2 \mathbb{Z} \longrightarrow 0,
$$

from which assertion 2 is a straightforward consequence. This completes the proof of Theorem 3.3.

By the actual construction of graphs $\Gamma_{\mathcal{B}}$ one can determine the possible quotient structures $M / M_{\mathcal{B}}$. For instance, the possible orders of cyclic quotients are the integers $a \leq \max \left(\frac{n-1}{2}, n-4\right)$ and moreover the even integers $b \leq 2(n-7)$. Apart from the usual exceptions in low dimensions, the cyclic structure does not provide the maximal index $\left[M: M_{\mathcal{B}}\right]$, which is equal to $5 \times 2^{\frac{n-9}{3}}, 6 \times 2^{\frac{n-10}{3}}$ or $7 \times 2^{\frac{n-11}{3}}$ according as $n$ is congruent to 0,1 or 2 modulo 3 (and equal to 2 for $n=5$ ).

A finer invariant for the module $M_{\mathcal{B}}$ is the set of vectors $v_{i}+v_{j}, i \neq j$ it contains.

Proposition 3.4. Let $\mathcal{B} \subset S$ be a basis for $E$ with graph $\Gamma=T \cup_{C \neq T} C$ reduced in the sense of Remark 2 , and excess $d$. Then the $\mathbb{Z}$-module $M_{\mathcal{B}}$ with basis $\mathcal{B}$ contains the vectors $v_{i}+v_{j}$ where $i$ and $j$ are distinct vertices 
in a same component and moreover at odd distance if this component is the tree. There are no other vectors $v_{i}+v_{j}$ in $M_{\mathcal{B}}$ except if $d=1$ or $d=2$ and $\Gamma$ is of even type, and then $M_{\mathcal{B}}$ also contains the $v_{i}+v_{j}$ for all vertices $i, j$ in the tree.

Proof. This follows directly from Propositions 2.4 and 2.5; we leave the details to the reader.

In particular, one can determine the maximal values of $s_{\mathcal{B}}=\left|M_{\mathcal{B}} \cap S\right|$ for a given dimension $n$. Apart from the case $M_{\mathcal{B}}=M$, i.e. when the reduced graph $\Gamma$ is a tree of excess 2 (and then $s_{\mathcal{B}}=\frac{n(n+1)}{2}$ ), the maximum value $s_{n}$ of $s_{\mathcal{B}}$ in a given dimension $n$ is attained when $\left[M: M_{\mathcal{B}}\right]=2$ : for $n=5,7,9, s_{n}=\frac{n^{2}+2 n-15}{4}(\Gamma$ is a tree of excess 4$)$; for $n \geq 7, s_{n}=\frac{n^{2}-7 n+24}{2}$ $(\Gamma=T \cup C$, where $T$ is a tree of excess 2 and of order 4 or $n-3$ ).

\section{Application to Coxeter lattices.}

In this section, $E$ is an $n$-dimensional Euclidean space of odd dimension $n \geq 5$. Recall that $\operatorname{Cox}_{n}$ is a scaled copy of $\mathbb{A}_{n}^{(n+1) / 2} \subset E$. As in the previous sections, $\mathbb{A}_{n}^{*}$ is generated by $n+1$ vectors $v_{0}, v_{1}, \ldots, v_{n}$ which add to zero. For a non-zero $x \in E$, let $p_{x} \in \operatorname{End}^{s}(E)$ be the orthogonal projection to the line $\mathbb{R} x$. Recall that a lattice $\Lambda$ with set of minimal vectors $S(\Lambda)$ is weakly eutactic if there exists in $\operatorname{End}^{s}(E)$ a linear relation

$$
\mathrm{Id}=\sum_{x \in S(\Lambda) / \pm 1} \rho_{x} p_{x}
$$

eutactic if there exists such a relation with strictly positive eutaxy coefficients $\rho_{x}$, and strongly eutactic if there exists such a relation with equal $\rho_{x}$ (which are then equal to $\frac{n}{s}>0$ ). This last condition amounts to saying that the set $S(\Lambda)$ is a spherical 2-design (or 3-design); see [Mar], Sections 3.1 and 16.1, and [Ven], Section 6.

An easy averaging argument shows that if $\Lambda$ is weakly eutactic, there exist systems of eutaxy coefficients which are constant on the orbits of the automorphism group of $\Lambda$. In particular, if the automorphism group of $\Lambda$ acts transitively on $S(\Lambda)$, weak eutaxy implies strong eutaxy.

The lattice $\operatorname{Cox}_{n}$ is strongly eutactic. Various strongly eutactic lattices related to $\operatorname{Cox}_{n}$ have been constructed in [B-M], using the equivariant Voronoi algorithm for the symmetric group $\mathfrak{S}_{n}$ or for its subgroup $\left(\mathfrak{S}_{m} \times \mathfrak{S}_{m}\right) \rtimes C_{2}, m=\frac{n-1}{2}$. Using such a deformation of the Euclidean structure, we obtained lattices denoted there by $C_{n}(n \geq 5)$ and $B_{n}(n \geq 5$ odd). In the scale which make them integral and primitive, $C_{n}$ has minimum $n-2(n$ odd $)$ or $2(n-2)$ ( $n$ even $)$, and $B_{n}$, a section of $C_{n+2}$, has minimum $n$. 
The two theorems below will allow us to recover the lattices above as cross-sections of Coxeter lattices having a much larger dimension.

Theorem 4.1. Let $n, p$ be integers with $n \geq 7$ odd and $3 \leq p \leq n-2$. Let $F_{n, p}$ be the span in $E$ of $v_{1}, \ldots, v_{p}$ and set $\operatorname{Cox}_{n, p}=\operatorname{Cox}_{n} \cap F_{n, p}$. The following conditions are equivalent:

(1) $\operatorname{Cox}_{n, p}$ is weakly eutactic.

(2) $\operatorname{Cox}_{n, p}$ is strongly eutactic.

(3) $p=\frac{n+3}{2}$.

[It results from [Ber] that for $p \geq 6$, the $p$-dimensional sections of $\operatorname{Cox}_{n}$ generated by minimal vectors whose kissing number is maximal (indeed, equal to $\frac{p(p-1)}{2}$ ) are the images of $\operatorname{Cox}_{n, p}$ under an automorphism of $\operatorname{Cox}_{n}$.]

Theorem 4.2. Let $n, p$ be integers with $n \geq 7$ odd and $3 \leq p \leq n-2$ odd. Let $F_{n, p}^{\prime}$ be the span in $E$ of the vectors $v_{i}+v_{j}, 1 \leq i, j \leq p, i$ odd, $j$ even, and set $\operatorname{Cox}_{n, p}^{\prime}=\operatorname{Cox}_{n} \cap F_{n, p}^{\prime}$. The following conditions are equivalent:

(1) $\operatorname{Cox}_{n, p}^{\prime}$ is weakly eutactic.

(2) Cox $_{n . p}^{\prime}$ is strongly eutactic.

(3) $p=\frac{n-1}{2}$.

Figure 3 below displays the graphs corresponding to the lattices $\operatorname{Cox}_{11,7}$ and $\operatorname{Cox}_{11,5}^{\prime}$ :
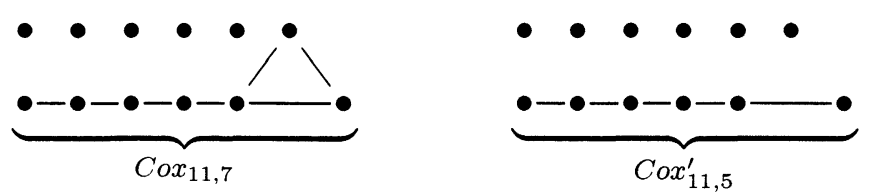

Figure 3

Proof. We shall only prove Theorem 4.1, leaving to the reader the proof of Theorem 4.2, which follows the same pattern.

For the proof, we work with $\mathbb{A}_{n}^{(n+1) / 2}$ (recall that $\operatorname{Cox}_{n}$ is a scaled copy of $\left.\mathbb{A}_{n}^{(n+1) / 2}\right)$. Its minimal vectors are the $\pm\left(v_{i}+v_{j}\right), i<j$, and it has minimum $m=\frac{2 n-2}{n+1}$. Due to the transitive action of $\mathfrak{S}_{p}$ on $S\left(\operatorname{Cox}_{n, p}\right)$, the assertions (1) and (2) are equivalent. To prove that (1) and (3) are equivalent, we evaluate $\sum_{1 \leq i<j \leq p} p_{v_{i}+v_{\jmath}}$. Taking into account the action of $\mathfrak{S}_{p}$, it suffices to evaluate this sum on $v_{1}$.

Using a Gram matrix for $\mathbb{A}_{n}^{*}$, it is not difficult to check that

$$
\begin{aligned}
\sum_{i<j} p_{v_{\imath}+v_{j}}\left(v_{1}\right) & =\sum_{1 \leq i<j \leq p} \frac{\left(v_{i}+v_{j}\right) \cdot v_{1}}{m}\left(v_{i}+v_{j}\right) \\
& =\sum_{2 \leq i<j \leq p} \frac{-2}{m(n+1)}\left(v_{i}+v_{j}\right)+\sum_{2 \leq j \leq p} \frac{n-1}{m(n+1)}\left(v_{1}+v_{j}\right) .
\end{aligned}
$$


Writing this sum as $\sum_{1 \leq k \leq p} a_{i} v_{i}$ and replacing $m$ by its value, we obtain

$$
\sum_{i<j} p_{v_{i}+v_{j}}\left(v_{1}\right)=\frac{p-1}{2} v_{1}+\frac{n-2 p+3}{2(n-1)} \sum_{2 \leq j \leq p} v_{j} .
$$

This formula clearly shows that $\sum_{i<j} p_{v_{i}+v_{j}}$ is proportional to the identity if and only if $n-2 p+3=0$.

Proposition 4.3. The lattice defined in Theorem 4.1 (resp. Theorem 4.2) is isometric to $C_{p}$ (resp. $B_{p}$ ).

Proof. We make use of the notion of a minimal class and its equivariant version as defined in [Mar], Sections 9.1 and 11.9.

The $\mathfrak{S}_{p}$-equivariant minimal class of the lattice $C_{p}^{\prime}=\operatorname{Cox} 2 p-3, p$ defined in Theorem 4.1 has dimension 1 , which shows that it is an equivariant Voronoi path, whose minimal vectors can be extracted from those of $\operatorname{Cox}_{n}$. In Theorem 4.3 of [B-M] it is proved that $C_{p}$ lies on an equivariant Voronoi path connecting $\operatorname{Cox}_{p}$ and the root lattice $\mathbb{D}_{p}$. This allows us to identify the $\mathfrak{S}_{p}$-equivariant minimal classes of $C_{p}$ and $C_{p}^{\prime}$. Since a minimal class contains at most one weakly eutactic lattice (up to similarity), the (strongly) eutactic lattices $C_{p}$ and $C_{p}^{\prime}$ are similar, hence isometric since they have the same minimum. (They are even $\mathfrak{S}_{p}$-isometric, i.e. isometric under an isometry which commutes with the action of $\mathfrak{S}_{p}$.)

The case of $B_{p}$ is dealt with by a similar argument.

Remark 4.4. In Theorem 4.1 , when $n=5$, we have $p=4$ and the lattice is only semieutactic (its eutaxy coefficients are non-negative, but some of them are zero). However, the 4-dimensional section having the largest possible kissing number is again a strongly eutactic lattice (similar to $\mathbb{A}_{2} \otimes \mathbb{A}_{2}$, with $s=9$ ).

Remark 4.5. The lattice $B_{p}$ can be also defined for any even $p \geq 6$. Then it is only semi-eutactic, but it nevertheless has only one non-zero eutaxy coefficient. In such a situation, the subset of its minimal vectors whose corresponding eutaxy coefficient is non-zero constitutes a spherical 2-design.

We could show that for $n \geq 7$ odd, the lattices $B_{(n-1) / 2}$ and $C_{(n+3) / 2}$ are the only weakly eutactic cross-sections of $\operatorname{Cox}_{n}$ having the same minimum as $\operatorname{Cox}_{n}$.

\section{References}

[Ber] A.-M. Bergé, On certain Coxeter lattices without perfect sections. J. Algebraic Combinatorics 20 (2004), 5-16.

[B-M] A.-M. Bergé, J. MARtinet, Symmetric Groups and Lattices. Monatsh. Math. 140 (2003), 179-195.

[Bo] B. BollobÁs, Modern graph theory. Graduate texts in Mathematics 184, Springer-Verlag, Heidelberg, 1998.

[Cox] H.S.M. Coxeter, Extreme forms. Canad. J. Math. 3 (1951), 391-441. 
[Mar] J. Martinet, Perfect Lattices in Euclidean Spaces. Grundlehren 327, Springer-Verlag, Heidelberg, 2003.

[Ven] B. Venkov, Réseaux et designs sphériques (notes by J. Martinet). In Réseaux euclidiens, designs sphériques et groupes, L'Ens. Math., Monographie 37, J. Martinet ed., Genève (2001), 10-86.

Anne-Marie BERgé

Université Bordeaux 1

351, cours de la Libération

33451 Talence Cedex, France

E-mail : berge@math.u-bordeaux1.fr

$U R L:$ http: //www.math.u-bordeaux.fr/ berge/

Jacques MARTINET

Université Bordeaux 1

351 , cours de la Libération

33451 Talence Cedex, France

E-mail : martinet@math.u-bordeaux1.fr

URL: http://www.math.u-bordeaux.fr/ martinet/ 